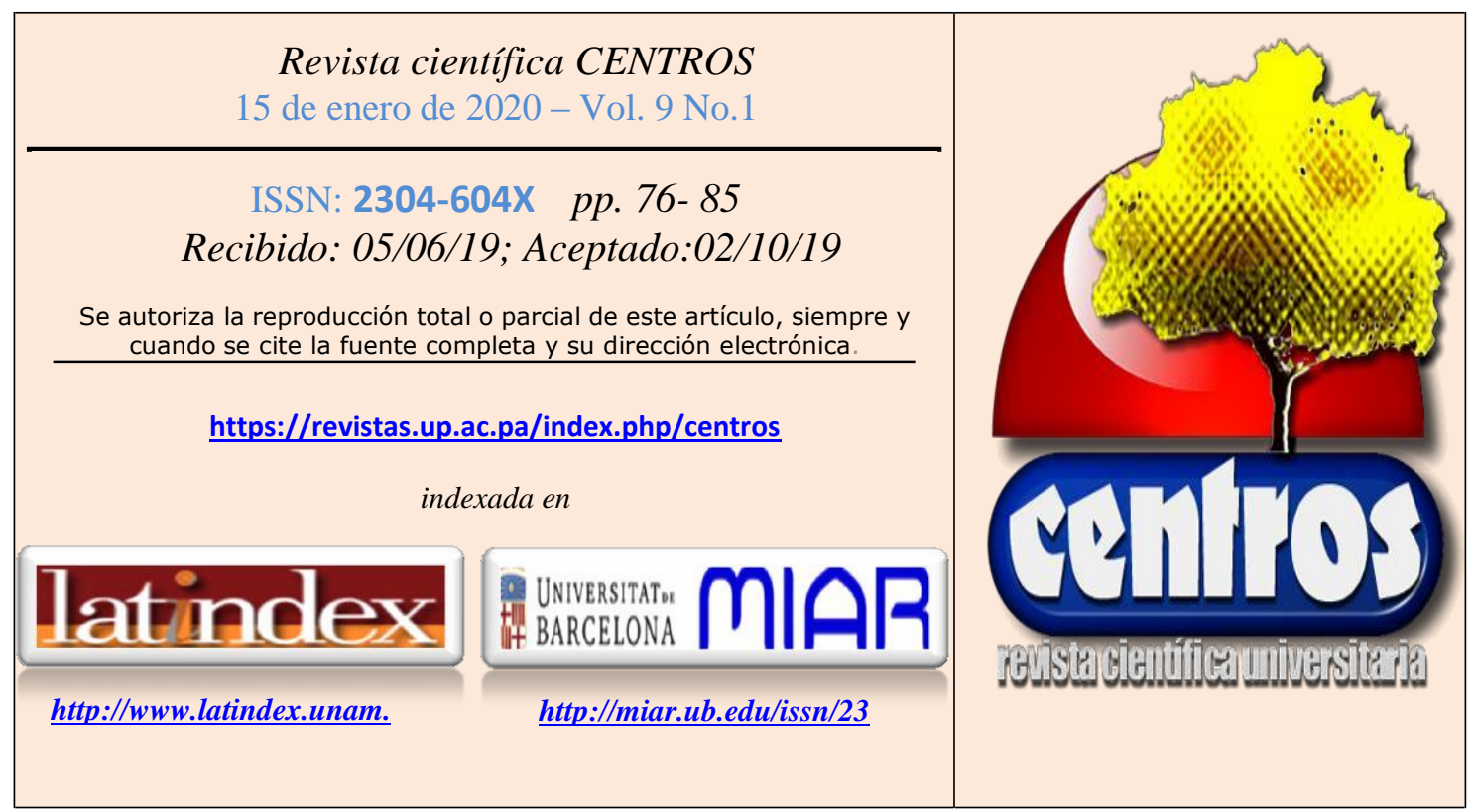

\title{
La ictioparasitología en la vinculación con la comunidad: un aporte a la producción piscícola con especies nativas en la provincia de Napo
}

Ichthyoprasitology in the connection with the community: a contribution to fish production with native species in the Province of Napo

Guerra P., Palma R., Vera A., Rodríguez-Haro C*

Universidad Regional Amazónica Ikiam. Cátedra de Parasitología. Grupo de Investigación de Microbiología Aplicada (MicApp). Km. 7, vía Muyuna, parroquía Muyuna. Tena. Ecuador. cerh81@yahoo.com.ar

\section{Resumen}

El cultivo de la "cachama" Piaractus branchypomus (Pisces: Characiformes), es una actividad que genera ingresos económicos en algunas personas de étnia Kichwa en la amazonía ecuatoriana. El objetivo del presente trabajo fue realizar actividades de vinculación con la ictioparasitología en los peces cultivados en las comunidades de San José y Santa Rita pertenecientes al Cantón Archidona, como parte de la colaboración de un convenio interinstitucional entre la Universidad Regional Amazónica Ikiam y la Fundación Centro Lianas, para fortalecer el cultivo piscícola mediante la vinculación. Se realizaron controles de ciertos parámetros físico-químicos en el agua (carbonatos, oxígeno disuelto), también se supervisó la calidad de la carne de la cachama y se evaluaron las condiciones sanitarias de los estanques. Se identificó la presencia de monogeneos con el 5,5\% de Prevalencia en peces juveniles y adultos colectados en la comuna San José y se hallaron quistes de protozoarios como las Myxosporas con $11,10 \%$ de Prevalencia, provenientes de los peces colectados en los estanques de la comunidad de Santa Rita. Además, se observó que los niveles de carbonatos son altos en los efluentes, en relación con los mostrados en otros estudios. Finalmente, se capacitó en el manejo técnico y sanitario del cutivo a los productores, se asistió, acompañó y recomendó acciones para reducir los resultados identificados.

Palabras clave: Parásitos, Cachama, Piaractus branchypomus, Gyrodactylidae, Myxosporidios. 


\section{Introducción}

Tanto en cultivos como en condiciones naturales, los peces son susceptibles al ataque de virus, bacterias, hongos y parásitos (Alcántara Bocanegra et al., 2015), en cultivos de altas densidades, pueden generarse condiciones negativas que debilitan su sistema immune con la probabilidad del ataque de patógenos (Verján, NRey \& Donado, 2001; Verján N, Iregui CA, 2015), también influyen los factores del medio ambiente (Carrillo, Aza, \& Nossa, 2007). Las infestaciones parasitarias tienen mayor impacto en la zona tropical (Franceschini et al., 2013).

Varios estudios han revelado que los parásitos son los agentes asociados a la mayor cantidad de muertes de Piaractus brachypomus. Las enfermedades parasitarias reportados en esta especie ictícola, han sido causadas por protozoarios Piscinoodinium sp., Ichthyophthirius multifilis y Trichodinas, Myxosporidios y con menor frecuencia por monogeneos (Verján, NRey \& Donado, 2001; Verján N, Iregui CA, 2015). La prevalencia de estos parásitos se han observado principalmente en las fases de alevinos y juveniles. Los signos causados por la infestación de estos parásitos varían según el agente causante. En un estudio realizado en cachamas blancas en Colombia, se reportó la infestación de estos peces con Piscinoodinium sp., siendo el causal de la enfermedad del terciopelo (Pádua, Ishikawa, Kasai, Jerônimo, \& Carrijo-Mauad, 2012; Theerawoot, 2008), esta enfermedad se caracteriza por la necrosis del epitelio lemelar, con pérdida de lamelas y la inflamación de filamentos branquiales. En el caso de infestación por $I$. multifilis, el cuadro patológico observado incluye hipertrofia y necrosis del epitelio lamelar (Dias \& Tavares-Dias, 2015; Poleo, Aranbarrio, Mendoza, \& Romero, 2011). En otros estudios, se han reportado que la presencia de parásitos estaría relacionada con un bajo control sanitario en el manejo (Franceschini et al., 2013; Verján N, Iregui CA, 2015).

La cachama, representa una fuente de ingresos económicos para algunas comunidades del cantón Archidona y en otras zonas de la amazonía ecuatoriana. La importancia del cultivo de esta especie radica en disminuir el comercio de especies invasoras fuertemente constituidas en la región como la tilapia y utilizar especies nativas con características de cultivo, la presencia de especies invasoras es una amenaza para las especies nativas y los ecosistemas acuáticos.

Por lo tanto, el estudio de la presencia de parásitos en este tipo de cultivos son una forma de controlar el correcto manejo de los peces y de asegurar su calidad para el consumo, su identificación, permite mejorar el manejo. Esto a su vez asegura la sostenibilidad de la actividad y su aplicación en otras comunidades. Por esto, el objetivo de este estudio fue identificar la existencia de parásitos, en caso de existir determinar la prevalencia parasitaria en los cultivos de cachama de las comunidades de San José y Santa Rita del cantón Archidona, provincia de Napo, Ecuador. Además, se planteó la evaluación de las condiciones físico-químicas del agua de los estanques de cultivo, es importante aclarar que en este proyecto se utilizó estos parámetros como indicadores de la calidad del agua y la salud del cultivo. Es decir, no se estudió las causas de la alteración de los parámetros, puesto que esto requiere una investigación más profunda, sino la relación del cambio en estos parámetros con la presencia o ausencia de parásitos y la identificación de parásitos como una alerta para posibles pérdidas económicas a las familias de las comunidades de San José y Santa Rita que se dedican a esta actividad piscícola.

\section{Antecedentes}

En las comunidades étnicas de la amazonía ecuatoriana, actualmente la práctica de la pesca, cacería y recolección combinada con la agricultura enfrentan problemas para abastecimiento de los alimentos, debido a los impactos negativos de actividades extractivas, 
monocultivos, ganadería e introducción de especies invasoras. Estas actividades ponen en riesgo la conservación del bosque y la biodiversidad de la región amazónica. El cultivo de la cachama representa para las comunidades indígenas amazónicas una fuente de recursos económicos y alimentos. La presencia de parásitos en las cachamas pueden afectar el crecimiento de los peces o infectar a humanos mediante la transmisión de parásitos zoonóticos presentes en la carne. Por lo que es necesario garantizar la seguridad de los consumidores y mejorar la eficiencia del proceso. Particularmente, en las comunidades de Santa Rita y San José (ubicadas en zonas cercanas al cantón Archidona, provincia de Napo), se alcanza hasta el momento una producción anual de $6.525,00 \mathrm{Kg}$ de carne de cachama blanca, de las cuales, el $58 \%$ es utilizada para autoconsumo y el $42 \%$ para la comercialización. A partir de esta actividad, se benefician 89 familias de manera directa (Almeida, 2017).

\section{Metodología}

El estudio se llevó a cabo en las comunidades de la etnia Kichwa de Santa Rita y San José, se analizaron 86 peces de diferentes estapas de crecimiento: 17 alevines, en las dos comunidades (Tabla 1). En la comunidad San José fueron 18 juveniles y adultos y en la comunidad de Santa Rita 51 individuos en etapa juvenil.Los peces fueron trasladados en bolsas con oxígeno hasta el laboratorio de Biología de la Universidad Regional Amazónica Ikiam, se colocaron en una pecera con oxigenación hasta su análisis. Los exámenes parasitológicos fueron para la identificación de ecto y endoparásitos, se analizaron la piel, aletas, ojos, branquias, cerebro, tejido muscular, órganos internos, intestinos, contenido estomacal, tejido hepático y frotis sanguíneos. Para los exámenes parasitológicos se utilizaron el estereoscopio Motic modelo SMZ-168® y se tomaron fotografías con el microscopio óptico Motic®.

Se realizaron análisis físico-químicos periódicos en las piscinas para oxígeno disuelto (OD), fosfatos, $\mathrm{pH}$, alcalinidad, dureza, temperatura y nitritos (Tabla 2), en la comunidad de Santa Rita se consideraron tres puntos de muestreo; el río que atraviesa la comunidad en la zona que está dentro de la reserva Colonso Chalupas, las piscinas de cultivo de peces de la comunidad de Santa Rita y el mismo río en la zona de descarga de agua de las piscinas y en la comunidad San José solo en las piscinas. Los análisis se realizaron en tres repeticiones, utilizando el kit para análisis de agua HI3817BP Hanna Instruments ${ }^{\circledR}$.

Finalmente, se efectuaron visitas técnicas, charlas sobre las Buenas Prácticas de Acuicultura BPA, para la prevención de parásitos y la divulgación de los resultados del estudio.

\section{Resultados}

\subsection{Alevines}

Las características morfológicas y parasitológicas de los alevines estudiados son expuestas en la Tabla 1.

Tabla 1. Datos de alevines y resultados de análisis.

\begin{tabular}{ll}
\hline \multicolumn{2}{c}{ Alevines } \\
\hline Individuos & 17 \\
Peso promedio & $28 \mathrm{mg}$ \\
Longitud promedio & $4.5 \mathrm{~cm}$ \\
Prevalencia parasitaria & $0 \%$ \\
\hline
\end{tabular}

Los alevines no presentaron presencia de parásitos. 


\subsubsection{Análisis de agua en alevines}

Los resultados de los análisis de agua indican que existe una posible fuente de contaminación, presumiblemente en el alimento que se suministra en los primeros días de cultivo. Otro factor a considerar es el transporte, las condiciones de los alevines provenientes del laboratorio, llegan en densidades de 500 individuos en cada bolsa con oxígeno. El estrés del transporte asociado con el alimento contaminado por possible almacenamiento deficiente, sumado a otros factores no identificados pueden favorecer a la proliferación de microorganismos dañinos como hongos filamentosos que pueden causar ulceraciones en la piel de los peces (Franceschini et al., 2013).

Tabla 2. Resultados análisis de agua en pecera de alevines.

\begin{tabular}{|c|c|c|c|c|c|c|c|}
\hline Variables & Control & M1 & M2 & M3 & M4 & M5 & M6 \\
\hline $\begin{array}{l}\text { Oxígeno disuelto } \\
(\mathrm{mg} / \mathrm{L})\end{array}$ & 11.4 & 9.5 & 6.8 & 1 & 9.5 & 9.7 & 7.7 \\
\hline Fosfatos (mg/L) & 0.2 & 1 & 0.8 & 1 & 1.5 & $>5$ & $>5$ \\
\hline Dureza (mg/L) & 10.6 & 13.5 & 16.5 & 9.3 & 8.7 & 7.8 & 8.4 \\
\hline \multicolumn{8}{|l|}{$\mathrm{CaCO} 3$} \\
\hline $\mathrm{pH}$ & 7 & 6.8 & 6.9 & 6.2 & 6.5 & 7.2 & 6.8 \\
\hline Temperatura ${ }^{\circ} \mathrm{C}$ & 24.2 & 23.8 & 23.8 & 22.4 & 22.4 & 23.6 & 23.6 \\
\hline Nitritos (mg/L) & 0.6 & 4.2 & 3.2 & 24.59 & 26.24 & 12.56 & 13.23 \\
\hline \multirow[t]{2}{*}{ Fecha de toma } & $17 / 06 / 20$ & $20 / 06 / 20$ & $20 / 06 / 20$ & $22 / 06 / 20$ & $22 / 06 / 20$ & $25 / 06 / 20$ & $25 / 06201$ \\
\hline & 18 & 18 & 18 & 18 & 18 & 18 & 8 \\
\hline
\end{tabular}

Los hongos y algas encontrados, se aislaron y fotografiaron y se muestran en las siguientes imágenes:

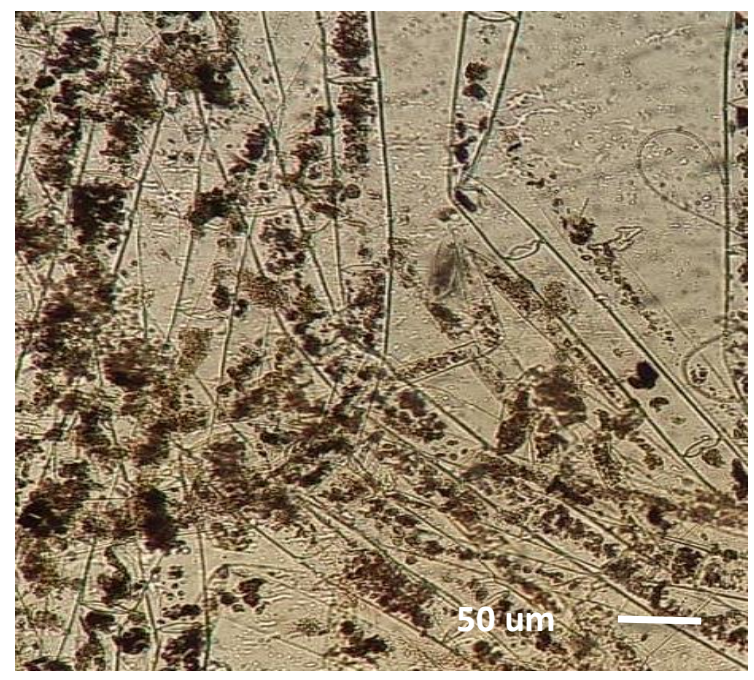

Figura 1. Esporangióforos no identificados presente en las muestras de agua. Microscopía óptica 10x.

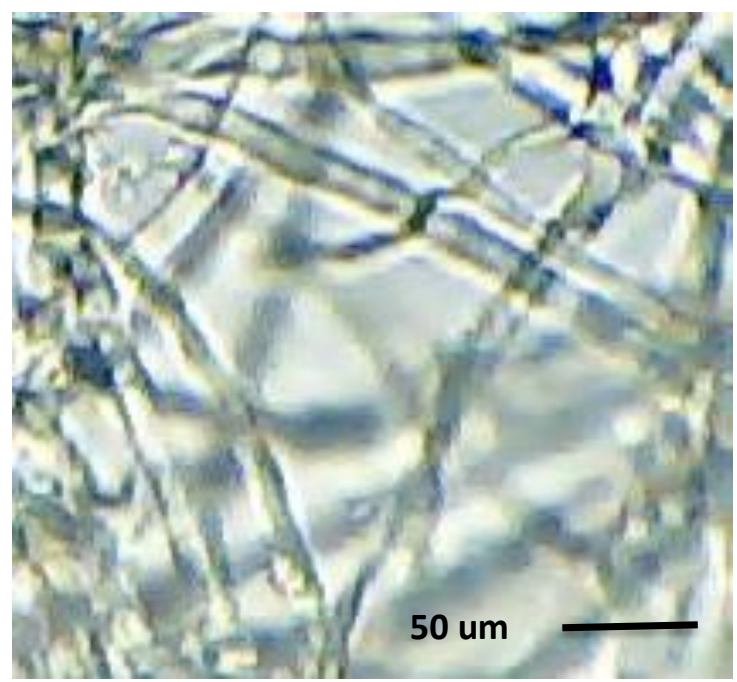

Figura 2. Algas en el agua. Se observan los pigmentos fotosintéticos. Microscopía óptica $40 \mathrm{x}$.

\subsection{Peces juveniles y adultos}


Los ejemplares muestreados de la Comunidad San José, fueron analizados y se encontraron en la piel un ejemplar de monogeneo juvenil perteneciente a la familia Gyrodactylidae (Figura $3)$.

Tabla 3. Datos de juveniles y adultos recolectados en la comuna San José.

\begin{tabular}{ll}
\hline & Juveniles y adultos \\
Individuos & 18 \\
Peso promedio & $2.82 \mathrm{~kg}$ \\
Longitud promedio & $20.7 \mathrm{~cm}$ \\
Prevalencia parasitaria Girodactylidae & $5,55 \%$ \\
Prevalencia parasitaria Myxosporidios & $11,10 \%$ \\
\hline
\end{tabular}

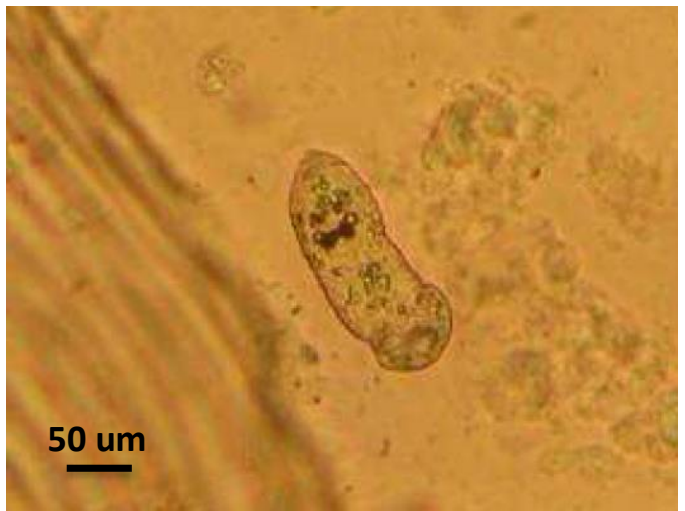

Figura 3. Monogeneo juvenil, familia Gyrodactylidae encontrado en superficie corporal. Microscopía óptica 40x.

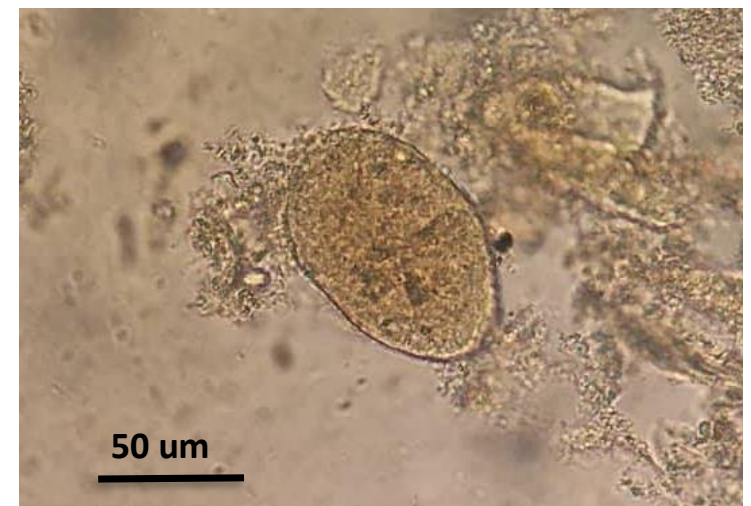

Figura 4. Quiste de Myxosporidios encontrados en intestino. Microscopía óptica $40 x$.

En la comunidad Santa Rita se identificaron Myxosporidios en la superficie del intestino (Figura 4). En los individuos de la piscina a cargo de la Unidad Educativa, no presentó presencia de organismos parasitarios.

Tabla 4. Datos de juveniles y adultos recolectados en la comuna Santa Rita.

\begin{tabular}{ll}
\hline \multicolumn{2}{c}{ Juveniles } \\
\hline Individuos & 51 \\
Peso promedio & $2.11 \mathrm{~kg}$ \\
Longitud promedio & $16.8 \mathrm{~cm}$ \\
Prevalencia parasitaria & $0 \%$ \\
\hline
\end{tabular}

\subsubsection{Análisis de agua}

En la Comunidad San José, los resultados obtenidos en este estudio (Tabla 5) indican que los parámetros de calidad del agua evaluados en las piscinas están dentro de los límites razonables de seguridad para la especie cultivada (Ingle de la Mora, Villareal Delgado, Arredondo Figueroa, Ponce Palafox, \& Barriga Sosa, 2003). 
Tabla 5. Resultados de análisis de agua en piscinas de la comunidad San José.

\begin{tabular}{cccc}
\hline Parámetro & Muestra 1 & Muestra 2 & Promedio \\
\hline $\mathrm{O}_{2}$ & $6,5 \mathrm{mg} / \mathrm{L}$ & $6,2 \mathrm{mg} / \mathrm{L}$ & $6,35 \pm 0,21 \mathrm{mg} / \mathrm{L}$ \\
Dureza & $11,1 \mathrm{mg} / \mathrm{L} \mathrm{CaCO} 3$ & $10,9 \mathrm{mg} / \mathrm{L} \mathrm{CaCO} 3$ & $11 \pm 0,14 \mathrm{mg} / \mathrm{L} \mathrm{CaCO} 3$ \\
Alcalinidad & $11,2 \mathrm{mg} / \mathrm{L} \mathrm{CaCO} 3$ & $11,1 \mathrm{mg} / \mathrm{L} \mathrm{CaCO} 3$ & $11,15 \pm 0,07 \mathrm{mg} / \mathrm{L}$ \\
& & & $\mathrm{CaCO} 3$ \\
Fosfato & $2,5 \mathrm{mg} / \mathrm{L}$ & $2,5 \mathrm{mg} / \mathrm{L}$ & $2,5 \mathrm{mg} / \mathrm{L}$ \\
Nitratos & $<10 \mathrm{mg} / \mathrm{L}$ & $<10 \mathrm{mg} / \mathrm{L}$ & $<10 \mathrm{mg} / \mathrm{L}$ \\
pH & 7,2 & 7,4 & $7,3 \pm 0,14$ \\
Temperatura & $22,3^{\circ} \mathrm{C}$ & $22,8^{\circ} \mathrm{C}$ & $22,55 \pm 0,35^{\circ} \mathrm{C}$ \\
\hline
\end{tabular}

La calidad del agua puede estar influenciada por varias condiciones que incluyen el alimento utilizado y la aireación que deben ser óptimas para evitar efectos fisiológicos adversos en los peces (Cai, Wermerskirchen, \& Adelman, 1996). El contenido estomacal de lo de los peces, indicaron que se alimentan de macroinvertebrados y vertebrados como ranas, lo que indica un ambiente sano para el crecimiento de las cachamas blancas.

En Santa Rita, al igual que los resultados de la comunidad de San José, los valores (Tabla 6) se encuentran dentro de los límites razonables de seguridad para la especie (Ingle de la Mora et al., 2003).

Tabla 6. Resultados de análisis de agua en piscinas de la comunidad Santa Rita.

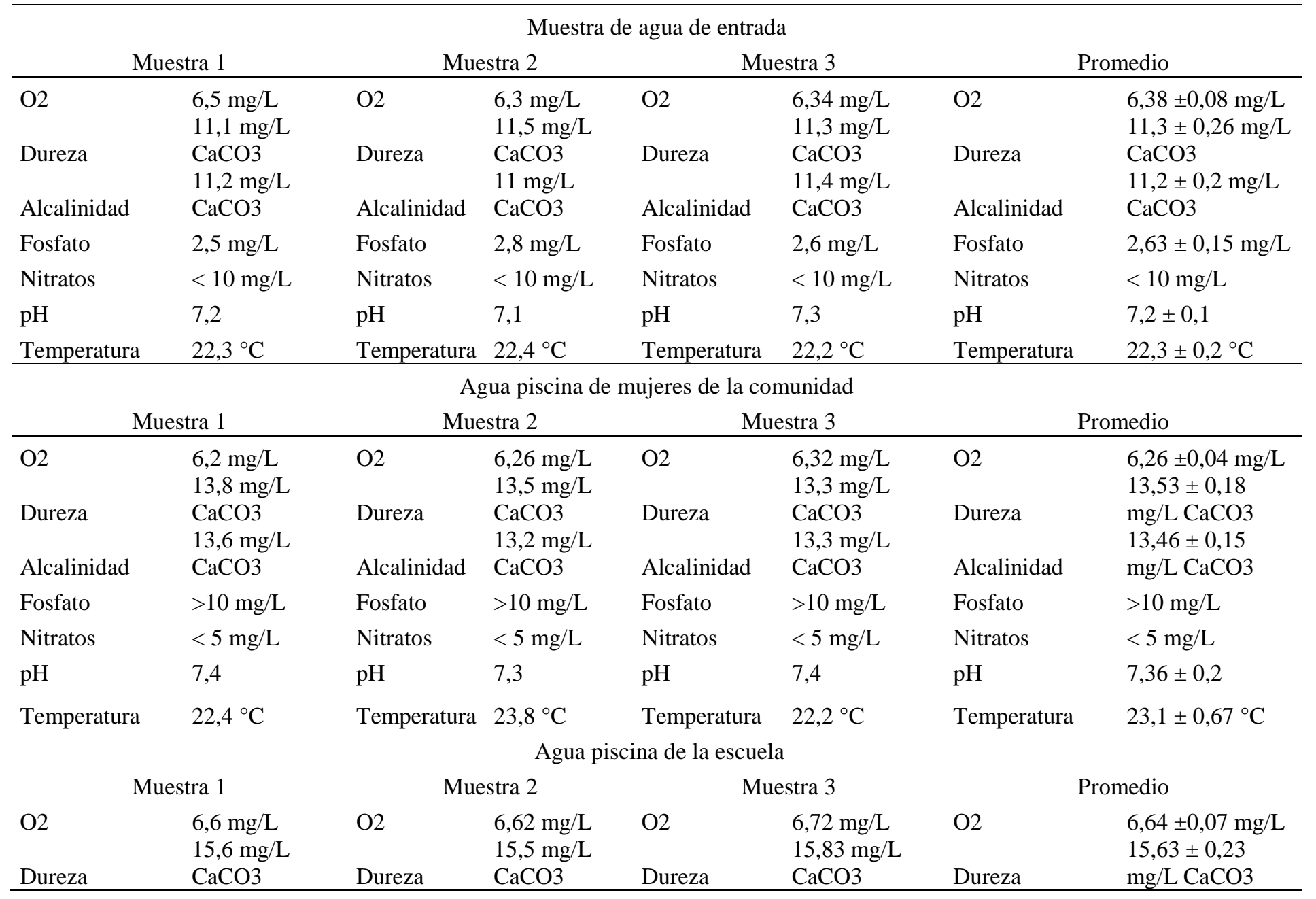




\begin{tabular}{|c|c|c|c|c|c|c|c|}
\hline Alcalinidad & $\begin{array}{l}16 \mathrm{mg} / \mathrm{L} \\
\mathrm{CaCO} 3\end{array}$ & Alcalinidad & $\begin{array}{l}15,72 \mathrm{mg} / \mathrm{L} \\
\mathrm{CaCO} 3\end{array}$ & Alcalinidad & $\begin{array}{l}15,8 \mathrm{mg} / \mathrm{L} \\
\mathrm{CaCO} 3\end{array}$ & Alcalinidad & $\begin{array}{l}15,84 \pm 0,31 \\
\mathrm{mg} / \mathrm{L} \mathrm{CaCO}\end{array}$ \\
\hline Fosfato & $>10 \mathrm{mg} / \mathrm{L}$ & Fosfato & $>10 \mathrm{mg} / \mathrm{L}$ & Fosfato & $>10 \mathrm{mg} / \mathrm{L}$ & Fosfato & $>10 \mathrm{mg} / \mathrm{L}$ \\
\hline Nitratos & $<10 \mathrm{mg} / \mathrm{L}$ & Nitratos & $<10 \mathrm{mg} / \mathrm{L}$ & Nitratos & $<10 \mathrm{mg} / \mathrm{L}$ & Nitratos & $<5 \mathrm{mg} / \mathrm{L}$ \\
\hline $\mathrm{pH}$ & 7,5 & $\mathrm{pH}$ & 7,6 & $\mathrm{pH}$ & 7,5 & $\mathrm{pH}$ & $7,55 \pm 0,2$ \\
\hline Temperatura & $22,8^{\circ} \mathrm{C}$ & Temperatura & $23,1^{\circ} \mathrm{C}$ & Temperatura & $23,2^{\circ} \mathrm{C}$ & Temperatura & $22,8 \pm 0,45^{\circ} \mathrm{C}$ \\
\hline \multicolumn{8}{|c|}{ Agua de salida de las piscinas } \\
\hline \multicolumn{2}{|c|}{ Muestra 1} & \multicolumn{2}{|c|}{ Muestra 2} & \multicolumn{2}{|c|}{ Muestra 3} & \multicolumn{2}{|c|}{ Promedio } \\
\hline $\mathrm{O} 2$ & $\begin{array}{l}5,9 \mathrm{mg} / \mathrm{L} \\
20,2 \mathrm{mg} / \mathrm{L}\end{array}$ & $\mathrm{O} 2$ & $\begin{array}{l}5,82 \mathrm{mg} / \mathrm{L} \\
20,5 \mathrm{mg} / \mathrm{L}\end{array}$ & $\mathrm{O} 2$ & $\begin{array}{l}5,87 \mathrm{mg} / \mathrm{L} \\
20,03 \mathrm{mg} / \mathrm{L}\end{array}$ & $\mathrm{O} 2$ & $\begin{array}{l}5,86 \pm 0,06 \mathrm{mg} / \mathrm{L} \\
20,2 \pm 0,3 \mathrm{mg} / \mathrm{L}\end{array}$ \\
\hline Dureza & $\begin{array}{l}\mathrm{CaCO} 3 \\
20,1 \mathrm{mg} / \mathrm{L}\end{array}$ & Dureza & $\begin{array}{l}\mathrm{CaCO} 3 \\
20,2 \mathrm{mg} / \mathrm{L}\end{array}$ & Dureza & $\begin{array}{l}\mathrm{CaCO} 3 \\
20,8 \mathrm{mg} / \mathrm{L}\end{array}$ & Dureza & $\begin{array}{l}\mathrm{CaCO} 3 \\
20,5 \pm 0,4 \mathrm{mg} / \mathrm{L}\end{array}$ \\
\hline Alcalinidad & $\mathrm{CaCO} 3$ & Alcalinidad & $\mathrm{CaCO} 3$ & Alcalinidad & $\mathrm{CaCO} 3$ & Alcalinidad & $\mathrm{CaCO} 3$ \\
\hline Fosfato & $5 \mathrm{mg} / \mathrm{L}$ & Fosfato & $5 \mathrm{mg} / \mathrm{L}$ & Fosfato & $5 \mathrm{mg} / \mathrm{L}$ & Fosfato & $5 \mathrm{mg} / \mathrm{L}$ \\
\hline Nitratos & $<5 \mathrm{mg} / \mathrm{L}$ & Nitratos & $<5 \mathrm{mg} / \mathrm{L}$ & Nitratos & $<5 \mathrm{mg} / \mathrm{L}$ & Nitratos & $<5 \mathrm{mg} / \mathrm{L}$ \\
\hline $\mathrm{pH}$ & 7,85 & $\mathrm{pH}$ & 7,70 & $\mathrm{pH}$ & 7,9 & $\mathrm{pH}$ & $7,82 \pm 0,1$ \\
\hline Temperatura & $23,8{ }^{\circ} \mathrm{C}$ & Temperatura & $22,9^{\circ} \mathrm{C}$ & Temperatura & $23,5^{\circ} \mathrm{C}$ & Temperatura & $23,4 \pm 0,39^{\circ} \mathrm{C}$ \\
\hline
\end{tabular}

Se debe destacar la diferencia en los valores de $\mathrm{pH}$ y alcalinidad del agua de salida. Existe un aumento en el pH y por lo tanto en la alcalinidad duplica su contenido en carbonatos, luego de su circulación en las piscinas. Estos resultados indican que el agua descargada no sería perjudicial para el afluente (Cai et al., 1996).

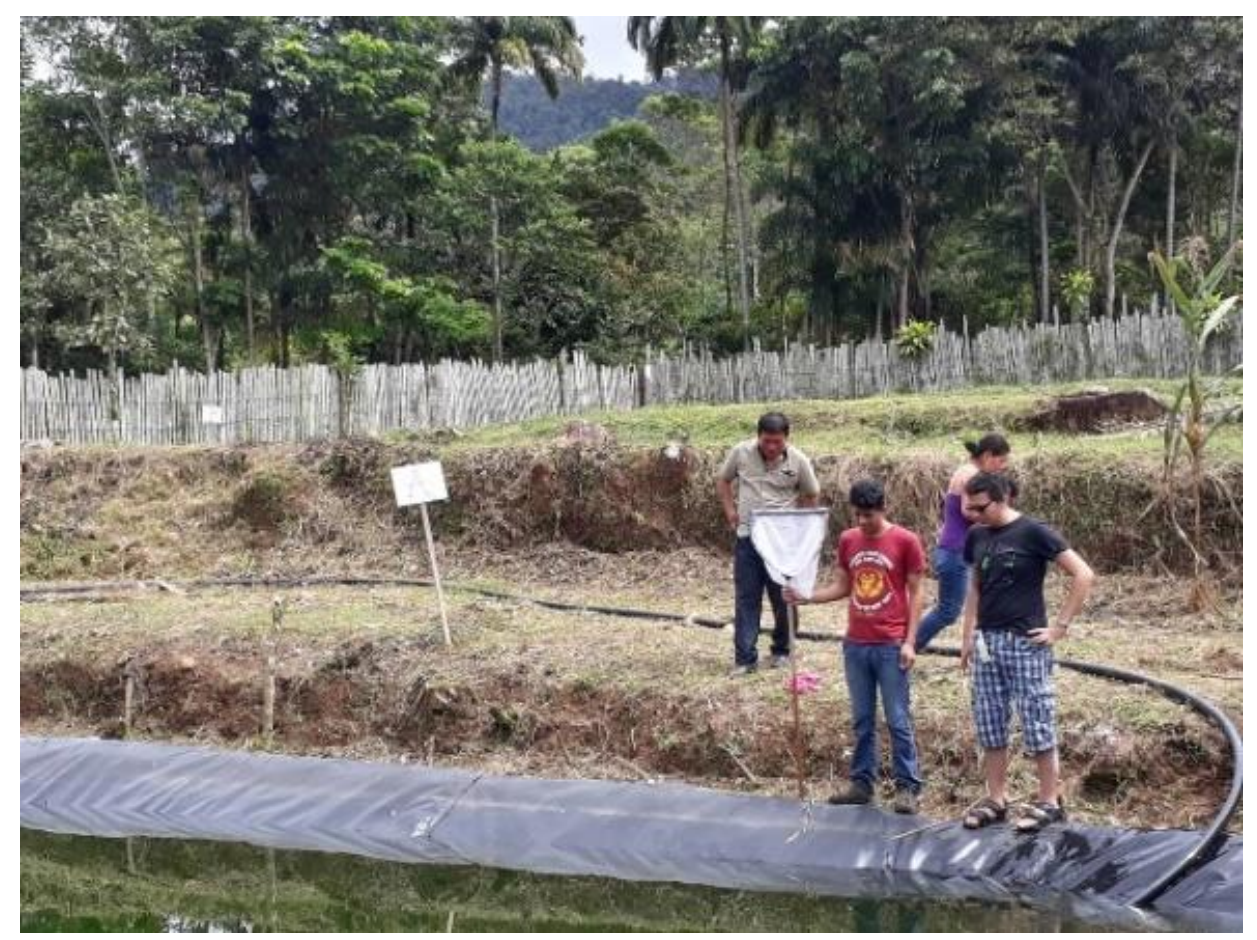

Figura 5. Fotografía tomada durante el muestreo realizado el 29/07/2018 en la comunidad de Santa Rita. 


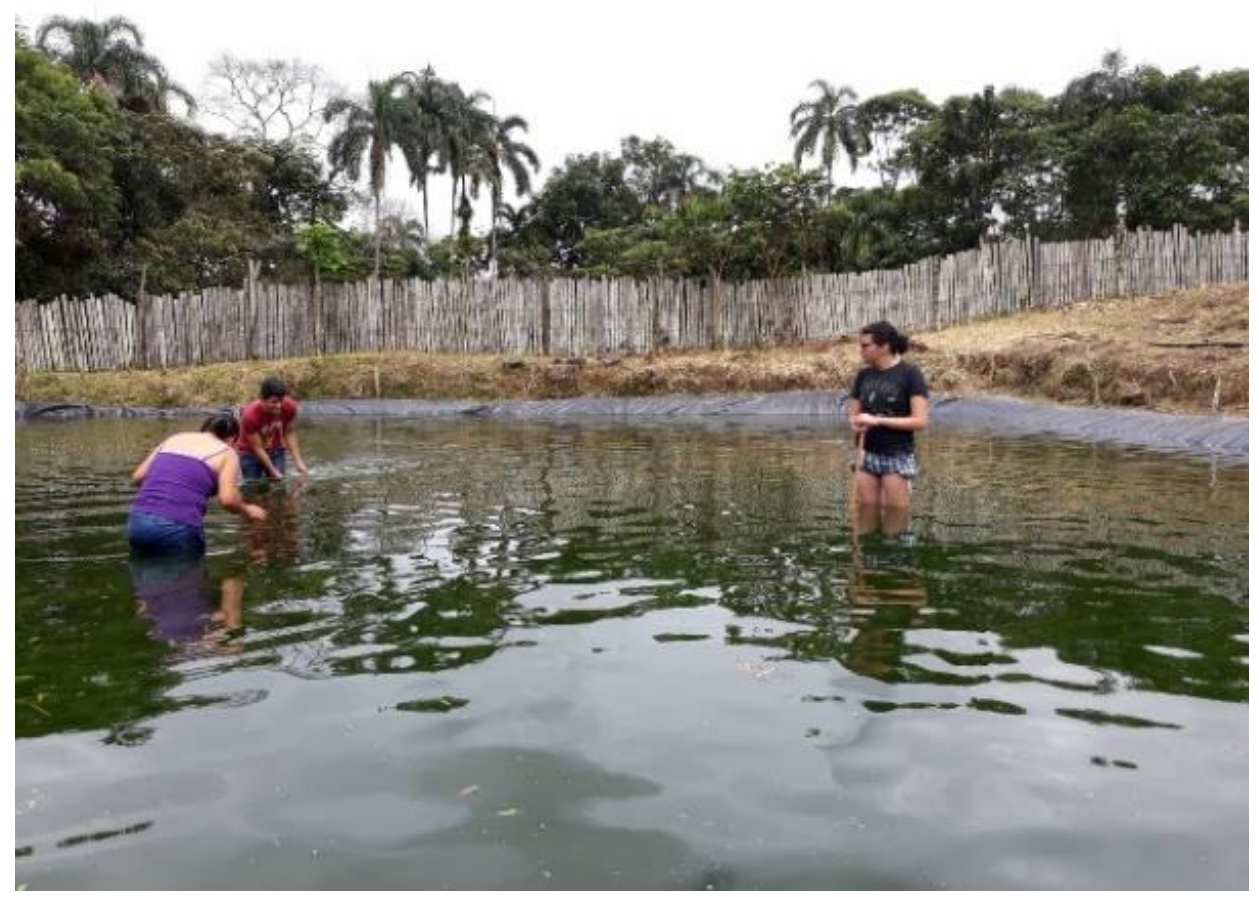

Figura 6. Fotografía tomada durante el muestreo realizado el 29/07/2018 en la comunidad de Santa Rita.

\section{Conclusiones}

El apoyo de la vinculación universitaria con las comunidades de la etina Kichwa en Santa Rita y San José del Cantón Archidona, Provincia de Napo, Ecuador, con actividades de asistencia técnica e investigación ha fortalecido la acuicultura con especies nativas.

Los estudios desarrollados para la identificación de parásitos mostraron presencia de monogeneos y myxosporidios, lo que permitió dar un soporte técnico como charlas para tomar medidas de control sanitario, por lo que se desarrollaron protocolos sencillos para aplicar al manejo piscícola. En general, los protocolos de manejo permitieron aplicar estas metodologías para las otras comunidades que realizan el cultivo de cachamas, practices como el uso de cal o ceniza, incrementar la aireación e identificar hospedadores intermediaros. Así también, realizer frecuentes análisis de control de calidad de agua, favorecieron en los niveles óptimos y deseados para el cultivo de cachama blanca. Además, los resultados indican que el agua descargada no sería perjudicial para el afluente.

Finalmente, el acompañamiento en asistencia técnica, establecer parámetros para el adecuado crecimiento de los peces que sirven como autoconsumo para las comunidades, lo cual ayuda a reducir el hambre y la desnutrición. Adicionalmente, asegura la continuidad del cultivo al disminuir la probabilidad de pérdidas asociadas a organismos parasitarios en la producción. Esto a su vez, permite la generación de fuentes de empleo y garantiza la sostenibilidad económica. Finalmente, la metodología de crianza implementada permite generar productos alimenticios con una huella ecológica reducida en comparación a otros sistemas de cultivo, fomentando la producción y el consumo responsable. 


\section{Recomendaciones}

Se recomienda implementar y desarrollar actividades de vinculación con otras comunidades que forman parte de la provincia de Napo y la amazónía, promover la piscicultura con especies naivas. Es necesario realizar control sanitario, inspecciones periódicas en zonas cercanas a las piscinas de cultivo para remover especies que pueden actuar como hospedadores intermediarios. Finalmente, se debería realizar análisis moleculares posteriores para confirmar la especie de los organismos parasitarios encontrados para poder implementar estrategias de control y remediación.

\section{Referencias}

Alcántara Bocanegra, F., Verdi Olivares, L., Murrieta Morey, G., Rodríguez Chu, L., Chu Koo, F., \& Del Águila Pizarro, M. (2015). Parásitos de alevinos de gamitana (Colossoma macropomum) y paco (Piaractus brachypomus) cultivados en el C.I. Quistococha, Loreto, Perú. Ciencia Amazónica (Iquitos), 5(1), 42. https://doi.org/10.22386/ca.v5i1.89

Almeida, A. (2017). Mujeres Indígenas y Piscicultura Nativa. (J. Robles Pilco, Ed.) (1st ed.). Quito: Fundación Centro Lianas.

Cai, Y. J., Wermerskirchen, J., \& Adelman, I. R. (1996). Ammonia Excretion Rate Indicates Dietary Protein Adequacy for Fish. Progressive Fish-Culturist, 58(2), 124-127. https://doi.org/10.1577/1548-8640(1996)058<0124

Carrillo, D. F. A., Aza, F. G. G., \& Nossa, M. O. (2007). Estudio ictioparasitológico de las especies cachama ( Piaractus brachypomus ) y mojarra roja ( Oreochromis spp .) en el Parque Ecológico “El Portal ”, municipio de Rionegro, Santander Ichtyoparasithological Study of the species Piaractus brachypomus an. Revista Spei Domus.

Dias, M. K. R., \& Tavares-Dias, M. (2015). Seasonality affects the parasitism levels in two fish species in the eastern Amazon region. Journal of Applied Ichthyology, 31(6), 1049-1055. https://doi.org/10.1111/jai.12865

Franceschini, L., Zago, A. C., Schalch, S. H. C., Garcia, F., Romera, D. M., \& da Silva, R. J. (2013). Parasitic infections of Piaractus mesopotamicus and hybrid (P. mesopotamicus X Piaractus brachypomus) cultured in Brazil. Revista Brasileira de Parasitologia Veterinária = Brazilian Journal of Veterinary Parasitology: Órgão Oficial Do Colégio Brasileiro de Parasitologia Veterinária, 22(3), 407-414. https://doi.org/10.1590/S198429612013000300015

Ingle de la Mora, G., Villareal Delgado, E. L., Arredondo Figueroa, J. L., Ponce Palafox, J. T., \& Barriga Sosa, I. de los A. (2003). Evaluation of some water quality parameters in a closed aquaculture recirculating-water system, submitted to diferent loads of fish. Hidrobiológica, 13(4), 247-253.

Klontz, G. (1991). Fish for the future: concepts and methods of intensive aquaculture.

Pádua, S. B. De, Ishikawa, M. M., Kasai, R. Y. D., Jerônimo, G. T., \& Carrijo-Mauad, J. R. (2012). Parasitic Infestations in Hybrid Surubim Catfish Fry. Revista Brasileira de Medicina Veterinária, 34(3), 235-240.

Poleo, G., Aranbarrio, J. V., Mendoza, L., \& Romero, O. (2011). Cultivo de cachama blanca en altas densidades y en dos sistemas cerrados. Pesquisa Agropecuaria Brasileira, 46(4), 429437. https://doi.org/10.1590/S0100-204X2011000400013

Theerawoot, L. (2008). Diversity and Distribution of External Parasites from Potentially Cultured Freshwater Fishes in Nakhonsithammarat, Southern Thailand. Diseases in Asian Aquaculture, 235-244.

Verján, NRey, I., \& Donado, P. (2001). Sistematización y caracterización de las lesiones 
branquiales de la cachama blanca ( Piaractus brachypomus ) de cultivo clínicamente sana: algunas interacciones hospedador-patógeno-ambiente . Revista AquaTic, 15, 1-15.

Verján N, Iregui CA, R. A. E. P. (2015). Estudio De Brotes De Enfermedad En La Cachama Blanca Piaractus Brachypomus : Diagnóstico Y Caracterización. Revista de La Facultad de Medicina Veterinaria y de Zootecnia, (JANUARY 2001). 